\title{
A presentation on EFECE: A new locking and compressing device with Kirschner-wire and a prelımınary study for bıomechanıcal evaluatıon of this system
}

emre Karadeniz ( $\nabla$ ekaradenizmd@gmail.com )

Research article

Keywords: Fracture Fixation, Internal Fixation; Bone Wires; Kirschner Wires; Distraction Force

Posted Date: January 13th, 2020

DOI: https://doi.org/10.21203/rs.2.20716/v1

License: (c) (1) This work is licensed under a Creative Commons Attribution 4.0 International License.

Read Full License 


\section{Abstract}

\section{Background}

This study aims to refine the biomechanical properties of a new Kirschner wire locking and compressing implant called EFECE.

\section{Methods}

EFECE implants are $5 \mathrm{~mm}$ in length, cylinder-shaped wire locking and compressing implants with an 8 $\mathrm{mm}$ radius and a hole for insertion of $1.2 \mathrm{~mm}$ Kirschner wire. The EFECE implant contains two pieces that are attached to each other with threads. The top piece functions as a cap, while the second piece contains three gloves for the insertion of magnetically active three balls. The locking mechanism is applied with the help of the balls in the cone-shaped gloves. The locking strength of these implants is determined through the following methods: for group 1, distraction force is applied to the devices until failure is reached, and then maximum force and total extension at the time of failure are evaluated; for group 2, cyclic distraction forces are applied to the implants against cyclic loading, and then the presence of failure and the maximum extension in a cycle are evaluated.

Results

In group 1, the mean pullout strength was 920 Newton, and the mean extension was $5 \mathrm{~mm}$. In group 2 there was no failure, and the mean maximum extension was $1.2 \mathrm{~mm}$.

\section{Conclusions}

EFECE implant holding power is sufficient for fracture stabilization. Our results show that these implants can be used for fracture fixation. The characteristics associated with this procedure (compression along Kirschner wires, freedome from bone quality fixation, percutaneous surgical technique, and the use of magnets for implant removal) may change surgical daily practice in the near future.

\section{Introduction}

Since the first operative treatment of bone fractures, medical science has been trying to improve the design of implants used in fracture treatment. Kirschner wire (k-wire) fixation, screw and/or plate fixation, intramedullary nailing, and external fixation are the main operative techniques used for fracture treatment currently. Each technique has advantages and disadvantages.

K-wire fixation does not generate compression force between bone fragments and K-wire migration, resulting in severe complications, which have been reported in the literature (1). During screw fixation, due to the core and thread diameter, small fragment fixation may be impossible. Screws for osteoporotic bone fracture fixation may not be able to get enough purchase between the fragments (2). Also, screw insertion 
is a time-consuming procedure for daily surgery. These challenges have prompted scientists to seek to improve existing and develop new fixation techniques.

The most appropriate fixation method depends on the bone, fracture type, patient's age, soft tissue condition, and surgeon's preference. Some complications that can accompany fracture treatment include infection, soft tissue irritation, implant exposure, and loosening, nonunion or malunion of the implant. Implant types and techniques are still evolving in search of the ideal option.

This study seeks to contribute to this evolution through the examination of the EFECE System for fracture fixation. The article presents a description of the EFECE and its use in fixation and compression of fractures with k-wires, and identifies its surgical kit, which is designed for percutaneous technique. The aim of this preliminary biomechanical study is to document the resistance of these EFECE implants on kwires against pullout forces and compare with similar studies in the literature.

\section{Methods}

The prototype of this device (EFECE, Micron Medical, Ankara, Turkey) is cylinder shaped with an $8 \mathrm{~mm}$ radius and a length of $5 \mathrm{~mm}$ and contains a hole for the insertion of $1.2 \mathrm{~mm}$ k-wire. The implant comprises two pieces that attach to each other with threads. The top piece functions as a cap, while the second piece contains three gloves for the insertion of three magnetically active metal balls, each with a $2.5 \mathrm{~mm}$ radius. The angle of the cone-shaped gloves to the k-wire axis is 7 degrees (Fig. 1). The presence of magnetically active balls in the locking mechanism enables removal of these implants with magnets; the magnetic force prompts the balls to move back to the base of the cone and release the k-wire. This implant removal technique presents an easy and new approach to orthopedic surgery.

The surgical kit for EFECE systems includes the sleeve, working cannula, screwdrivers, k-wire tensioner, wire cutter, and magnet. The surgical kit is designed for a percutaneous surgical technique. After reduction of the fracture and $\mathrm{k}$-wire placement conducted through conventional techniques, two reciprocal EFECE implants are pushed forward on k-wires until bone cortex contact is achieved with the help of sleeve, working cannula, and screwdrivers. Compression force can be applied on the fractured fragment with the help of the k-wire tension band. The remaining part of the k-wire is cut with a percutaneous wire cutter. At this stage, there is no need to rotate the screwdriver on the K-wire and there is no need to measure the diameter of the implant, which are time-consuming procedures for screw insertion.

When implants are locked with screwdrivers, the devices do not allow forward or backward movement on k-wires. EFECE implants allow only forward movement on k-wires. After insertion of k-wires across a fracture line, EFECE implants lock the k-wire against backward forces. When pulling back, with the help of the locking mechanism, implants catch the k-wire, preventing backward movement (Fig. 2). Compression force can be applied to the fracture line over these implants with implant advancement and k-wire tensioning. After locking these implants, k-wire and the device system hold the fractured fragments in the desired position. 
The EFECE implant locking mechanism is helped by the balls in the cone-shaped gloves. In forward movement, the balls move back to the base of the cone and release the k-wire. During pulling back, balls move to the narrow part of the cone and lock the k-wire. (Fig. 1)

To define the locking strength of these EFECE implants, in group 1, devices were placed on $1.2 \mathrm{~mm}$ stainless-steel k-wires (Sayan Medical, Izmir, Turkey) and tightened. With the help of jaws, the implant and k-wire system are attached to the Lloyd Instruments LR 50K Universal Materials Testing Machine (Lloyd Instruments Ltd., Hants, United Kingdom) (Fig. 3). An increasing distraction force of $2 \mathrm{~mm} / \mathrm{min}$ is applied until failure. The maximum force and the total extension at the time of failure are then evaluated with the help of Nexygen software (Fig. 4).

To define the behavior of these EFECE implants against cyclic loading, in group 2, implants are placed on $1.2 \mathrm{~mm}$ stainless-steel k-wires (Sayan Medical, Izmir, Turkey) and tightened. With the help of jaws, the device and k-wire system are attached to the Lloyd Instruments LR 50K distraction machine (Fig. 3). Then 100 cyclic distraction forces of 450 Newton, $10 \mathrm{~mm} / \mathrm{min}$ are applied. The presence of failure and the maximum extension in a cycle are then evaluated with the help of the Nexygen software (Fig. 5).

\section{Results}

In group 1, after 10 experiments, the mean pullout strength was 920 Newton (range: 837-1026 N), and the extension was $5 \mathrm{~mm}$ in all experiments. In all experiments in group 1, failure occurred between the balls and the k-wire. K-wires were crushed with the balls, and there was no failure of the k-wire.

In group 2, after 10 experiments, there was no failure of the EFECE implant. Each implant resisted against $450 \mathrm{~N}$ of 100 cyclic distraction forces. The mean maximum extension was $1.2 \mathrm{~mm}(0.41-2.42 \mathrm{~mm})$, and the mean first cycle extension was $1.07 \mathrm{~mm}(0.36-2.31 \mathrm{~mm})$.

\section{Discussion}

Using the new patented EFECE system, metaphyseal fractures can be treated with an internal fixation technique. Internal fixation techniques developed in the surgical treatment of metaphyseal fractures until today are as follows: K-wire, screw, plate-screw and the intramedullary nail. In the techniques, the aim is to keep fracture fragments in the desired position until healing of the fracture has occurred. Advantages and disadvantages of these techniques vary by fracture types and influence the treatment plan of the surgeon.

In fixation with k-wires, after reduction of the fracture, fragments are fixed by passing these straight wires through the bone fragments. The most important restriction of this technique is the forward/backward movement of the fractured fragments along the wire, causing malunion or nonunion (4). After reduction, these wires do not provide compression between the fracture fragments. In addition, these wires may migrate after they are placed into the bone $(5,6)$. In the literature, there are designs to solve this problem, such as pinballs and pin rubbers. However, these devices are designed for external usage; they are not 
suitable for k-wire internal fixation. In this study we show that when using the EFECE system, the mean kwire holding strength of the new implant is 920 Newton. This is enough holding power to prevent the kwire migration problem, and bone quality does not play a role.

Screw insertion is a time-consuming procedure. In fixation of fractures with screws, first the fractured fragments are reduced, and then screws are inserted to hold fragments in the desired position. In the working mechanism of these screws, compression is obtained between fragments on the basis of creating mutual forces between the screw head and the screw threads (7). In the screw insertion technique, the size of the screw can be increased to increase compression force and fixation strength between the fragments. Increasing the size of the screw may not be possible when bone fragments are small. Due to the size of the screws, there may not be enough places between the fragments to insert additional screws. In the surgical technique using conventional screws, first the bone is drilled for screw core by means of a drill, then tapping is performed for screw threads, and afterward the screws are inserted. Before these procedures, sometimes a k-wire is placed in order to additionally guide the cannulated screw. The conventional screw surgical technique is somewhat difficult, and the fixation provided by means of the screw threads may not be possible for patients with osteoporosis. For medial malleol fixation with screws, Polland JD et al. reported the pullout strength of two 3.5-mm fully threaded bicortical screws to be $327.6 \mathrm{~N}$ (range 117.5 to $804.3 \mathrm{~N}$ ) (8). the EFECE system does not require rotation of the screwdriver on the k-wire. In the same study, unicortical, partially-threaded two cancellous screw resistance to pullout forces was $116.2 \mathrm{~N}$ (range 70.2 to $355.5 \mathrm{~N}$ ). In our study, the pull-out resistance of the Kirschner wire-EFECE interface is 920 Newton, and there is no role of bone quality for this purchase. In our newly defined technique, the fixation is with at least two reciprocal EFECE devices and a k-wire. The fixation strength is not affected by bone quality; the holding power of this system is achieved through counter compression and tightening of these devices on the k-wire. Additionally, no rotary motion is required for screwing, which is a time-consuming period in conventional orthopedic surgery techniques.

The literature contains reports comparing pullout resistance of fixation techniques. Schlitz et al. biomechanically compared screw and k-wire fixation for lateral condyle fractures (9). They apply compression and distraction force after fixation with 2 divergent k-wires or a lag screw. The average maximum tension force for the screw fixed sample was $110.8 \mathrm{~N}$ compared with $24.7 \mathrm{~N}$ with k-wire fixation. As a conclusion, they reported that screw fixation provides increased biomechanical stability of the construct compared to k-wires.

In fixation of fractures with plate and screws, after reduction, fracture fragments are fixed by means of plates attached via screws. However, because plate attachment is performed by means of screws, disadvantages in the application of screws also exist. Plate placement requires more peripheral soft tissue to be stripped, which results in more incision and more vascularization problem for bone fragments (10). The plate may hinder skin closure in areas with thin subcutaneous tissue, and plates may be palpable. Plate placement may not be possible when bone fragments are small or close to the joint. Additionally, after fracture treatment, bone and peripheral tissues are re-damaged during plate removal, 
causing well-defined complications (11). With two EFECE devices and a Kirschner wire, we can compress and fix the fractured fragments.

In 2001 Saeki et al. defined a new fixation technique using curved titanium pins, and they compare this new technique (NODE Anchoring System) to conventional k-wire techniques using three different configurations (12). They fix the Colles' fracture with NODE Anchoring System, using ball tipped pins inserted obliquely through the dorsoradial shaft of the radius. Wedge-shaped stoppers are impacted into the proximal entry portals to prevent pin migration. Then they apply compression force on the articular surface. They concluded that the load at failure was significantly greater for NODE System than for k-wire models.

EFECE implants may function as a k-wire fixation system for small and close to the joint line fracture fragments because fixation and compression can be achieved through use of the thin k-wires, compared to the conventional use of screws in daily practice. Also, it can function as a screw for compression of the fracture fragments over k-wires. With this new fixation technique, fracture of difficult anatomic locations for screw placement, like an elbow, may be treated with thin k-wires by compression.

Fixation and compression can be achieved independently from bone density with these implants. Balls in the implant compress the k-wire after locking the device, preventing forward or backward movement. There is no role of the bone in this locking mechanism. The fixation system of these devices may function as a "bag of bones," which would make it a new option suitable for osteoporotic patients.

Systems reconstructed with two of these devices and a k-wire may function as a more effective buttress in fracture treatment that than the buttress plate conventionally used in medial plateau fracture. Placing these devices across the fracture line to compress and hold the fractured fragment against inferior slip means simple and minimal surgery.

For patellar fracture surgical treatment, anterior tension band wiring currently gives the best results, with $85 \%$ of patients reporting good or excellent outcomes according to 2 studies providing a combined cohort of 59 patients $(13,14)$. However, the high occurrence of symptomatic fixation implants has spurred the development of novel constructs. With our newly defined fixation system, because the implants are only $8 \mathrm{~mm}$ in radius and $5 \mathrm{~mm}$ in length, it is theorized that symptomatic implant complications will occur less frequently.

The mean pullout force that can be applied to these implants was 920 Newton after locking (range: 837$1026 \mathrm{~N}$ ). This value is enough for resisting distraction forces across the k-wire during fracture treatment. Placing two or more k-wires at different angles and compressing the fracture line with these devices may resist too much more force.

The mean extension of $5 \mathrm{~mm}$ during distraction force was mostly due to the slip of the balls on k-wires. The ratio of k-wire lengthening during pulling forces and its total value of contribution to total extension is a topic for another study. 
In our study, against $450 \mathrm{~N}$ of 100 cyclic distraction force, no implant failures occurred. This means no severe ball/Kirschner wire or ball/device's inner surface friction force reduction during cyclic loading. The mean maximum extension was $1.2 \mathrm{~mm}$, and the mean first cycle extension was $1.07 \mathrm{~mm}$. We were not able to locate data with which to compare with conventional techniques.

There are limitations of EFECE systems. EFECE systems require at least two reciprocal EFECE implants for a k-wire fixation. The counterpart of the k-wire that goes through the fragments and leave the bone cortex needs to be prepared for EFECE insertion. With the EFECE system, the fixation strength is dependent upon the mechanical properties of a thin k-wire.

There are limitations in this study. Testing along a single axis cannot appropriately simulate physiological loading at the fracture site. Because this is a new technique, information on the amount of compression force that can be applied with the help of wire tensioning is not available. These unknown data will examined in the next study.

More study is needed to confirm the efficacy of EFECE systems as fixation techniques due to the newness of the procedure. However, these results provide initial validation of EFECE Systems as an effective new implant technology for the repair of fractures.

\section{Conclusions}

This study is the first step in the establishment of EFECE systems as a new fixation technique in orthopedic surgery that produces better results using minimally invasive surgical effort. If the EFECE Systems do so; small bone fragments, osteoporotic bone fractures, difficult anatomic locations for screw placement may be treated more easily with these new implants. Moreover, the implant removal with magnets will make our implant removal technique simpler and minimally invasive.

\section{Abbreviations}

K-wire: Kirschner wire

$\mathrm{N}$ : Newton

Mm: Millimeter

\section{Declarations}

Ethics approval and consent to participate: Not applicable

Consent for publication: Not applicable

Availability of data and materials: All data generated or analysed during this study are included in this published article 
Competing interests: The authors declare that they have no competing interests

Funding: No funding has been recieved for his research.

Authors' contributions: Emre Karadeniz is the patent owner, study designer and writer of this research. The author read and approved the final manuscript.

Acknowledgements: Not applicable

\section{References}

1. Bezer M, Aydin N, Erol B, Laçin T, Güven O. Unusual migration of K-wire following fixation of clavicle fracture: a case report. Ulus Travma Acil Cerrahi Derg. 2009;15(3):298-300.

Cornell CN. Internal fracturefixationin patients with osteoporosis. J Am Acad Orthop Surg. 2003;11(2):109-19.

2. Emre Karadeniz (2011, Oct 17). efece video 1, video, https://m.youtube.com/watch?v=AloRcFcae6g

3. Hsu LP, Schwartz EG, Kalainov DM, Chen F, Makowiec RL. Complications of K-wire fixation in procedures involving the hand and wrist. J Hand Surg Am.2011;36(4):610-6.

4. Tan L, Sun DH, Yu T, Wang L, Zhu D, Li YH. Death Due to Intra-aorticMigration of Kirschner Wire From the Clavicle: A Case Report and Review of the Literature. Medicine. 2016;95(21):e3741.

5. Eroğlu M, Özcan Ö, Şentürk I, Yücehan M. An extraordinary mechanism causing intraoperativemigration of the Kirschner-wire. Eklem Hastalik Cerrahisi. 2016;27(1):58-60. Hughes AN,Jordan BA. Themechanical properties of surgical bone screws and some aspects of insertion practice. Injury. 1972;4(1):25-38.

6. Pollard JD,Deyhim A,Rigby RB, Dau N, King C, Fallat LM, Bir C. Comparison of pullout strength between 3.5-mm fully threaded, bicortical screws and 4.0-mm partially threaded, cancellous screws in the fixation of medial malleolar fractures J Foot Ankle Surg. 2010;49(3):248-52.

7. SchlitzRS,Schwertz JM, Eberhardt AW, Gilbert SR. Biomechanical Analysis of Screws Versus K-Wires for Lateral Humeral Condyle Fractures.J Pediatr Orthop. 2015;35(8):e93-7.

8. Melamed E,Joo L,Lin E, Perretta D, Capo JT. Plate Fixation versus Percutaneous Pinning for Unstable Metacarpal Fractures: A Meta-analysis J Hand Surg Asian Pac Vol. 2017;22(1):29-34.

9. Langkamer VG, Ackroyd CE. Removal of forearm plates. A review of the complications. J Bone Joint Surg $\mathrm{Br}$ 1990; 72: 601-4.

10. SaekiY,Hashizume H, Nagoshi M, Tanaka H, Inoue H. Mechanical strength of intramedullary pinning and transfragmental Kirschner wire fixation for Colles' fractures J Hand Surg Br. 2001;26(6):550-5.

11. Levack B, Flannagan JP, Hobbs S. Results of surgical treatment of patellar fractures. J Bone Joint Surg $\operatorname{Br} 1985 ; 67(3): 416-9$.

12. Bostman O, Kiviluoto O, Santavirta S, Nirhamo J, Wilppula E. Fractures of the patella treated by operation. Arch Orthop Trauma Surg 1983;102(2):78-81. 


\section{Figures}

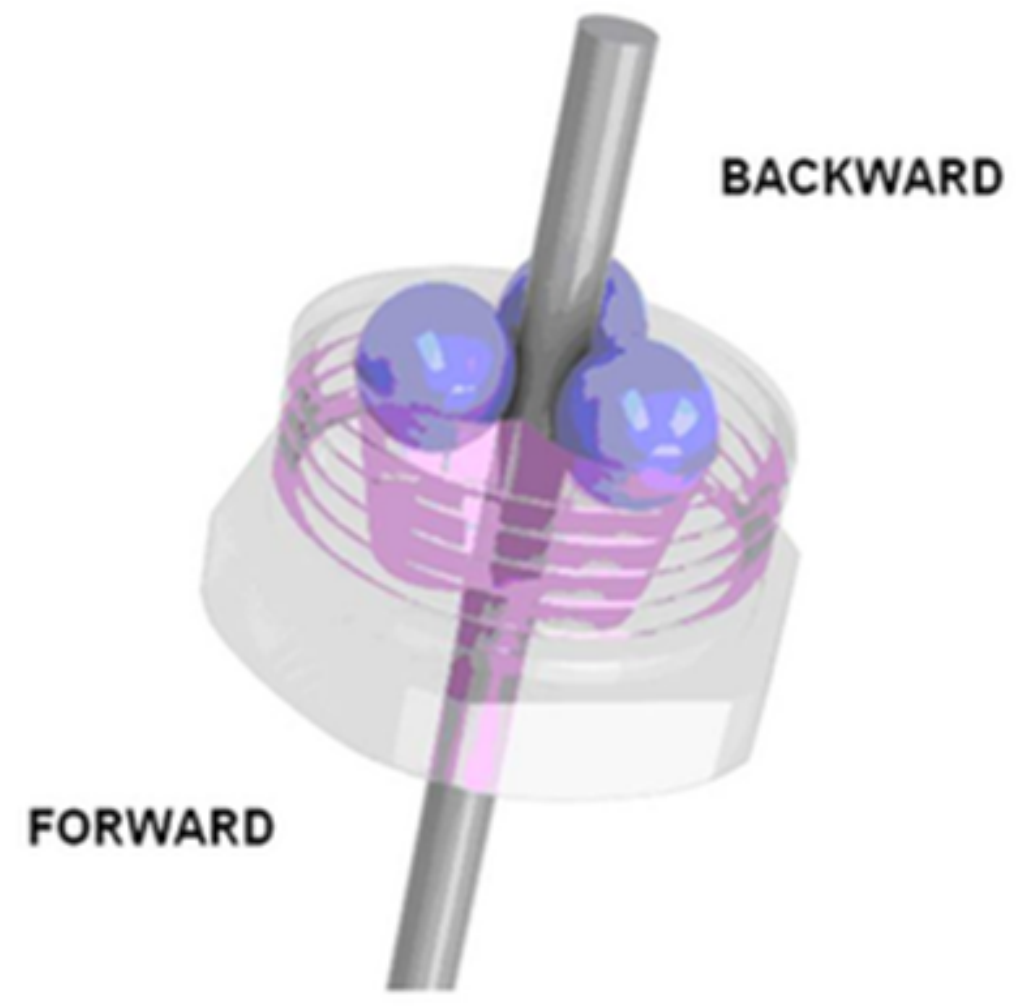

Figure 1

EFECE implant's locking mechanism is with the help of metal balls in cone-shaped gloves. For the forward movement to compress the fractured fragments across the k-wire, balls move back to the base of the cone and release the $k$-wire. During pulling back, balls move to the narrow part of the cone and lock the k-wire. 


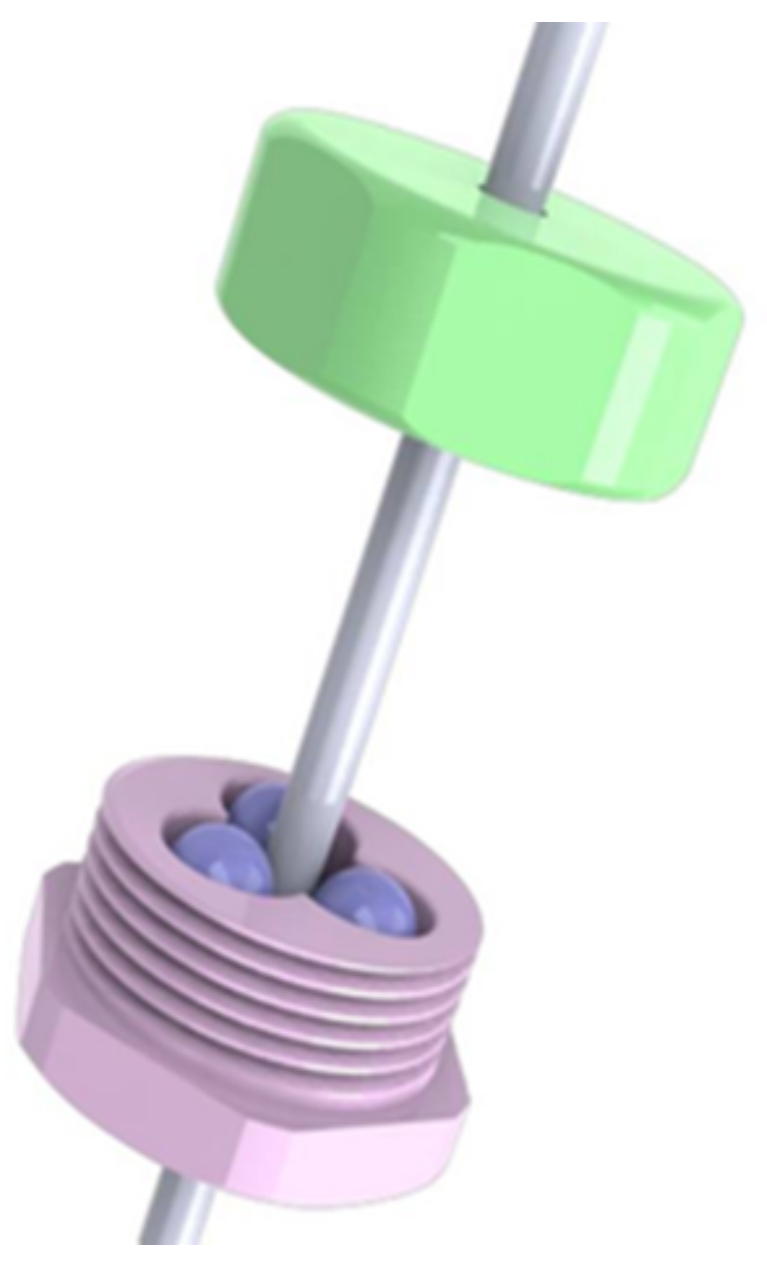

Figure 2

The prototype of this EFECE implant contains two pieces that catch each other with threads. The top piece functions as a cap. The second piece contains 3 gloves for the insertion of 3 metal balls. These balls are with $2.5 \mathrm{~mm}$ radius and magnetically active. The cone-shaped gloves angle to the k-wire axis is 7 degree. There is a hole for the insertion of $1.2 \mathrm{~mm}$ k-wire at each piece. 


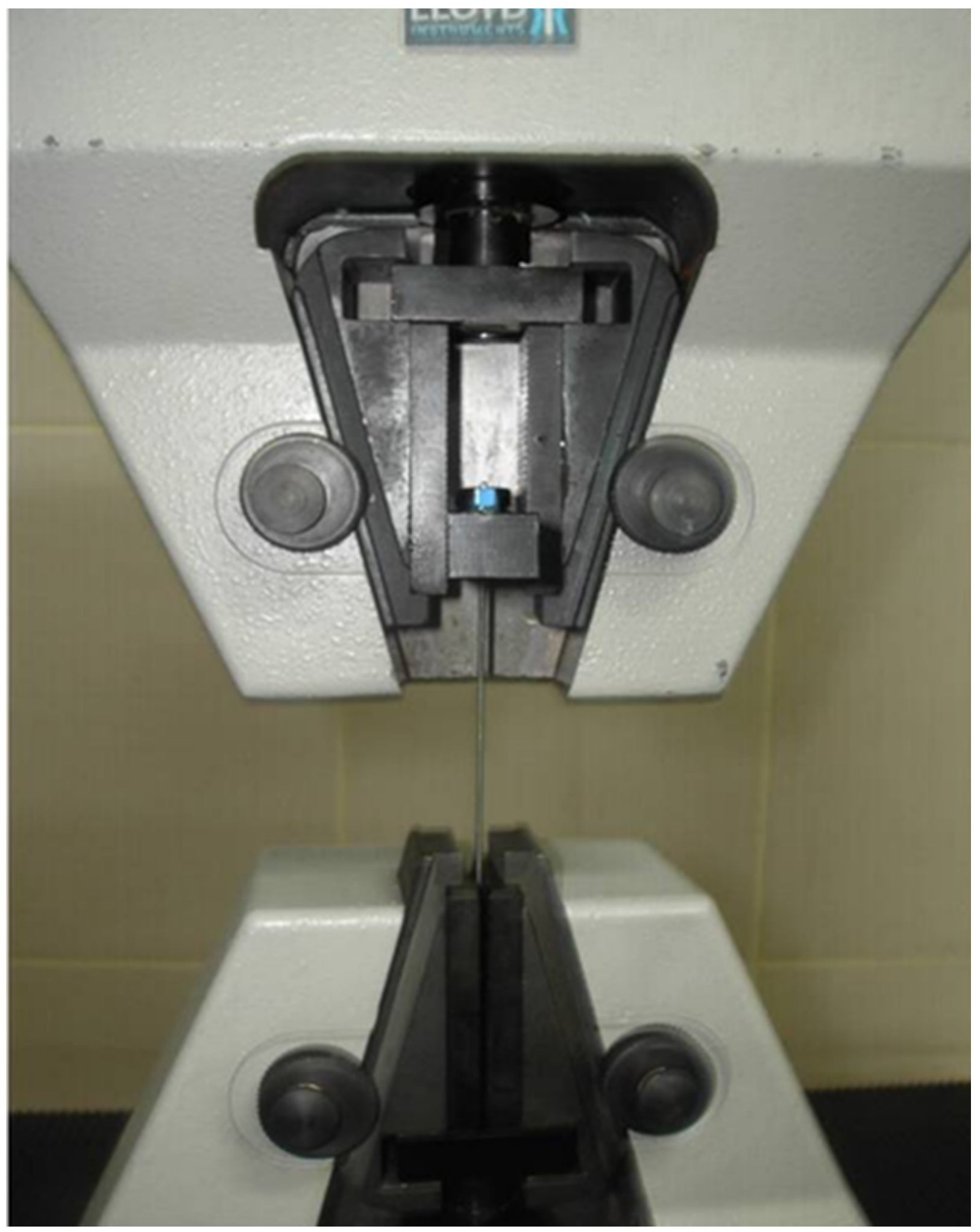

Figure 3

EFECE implant and k-wire system attached to the Lloyd Instruments LR 50K Universal Materials Testing Machine with the help of jaws and increasing distraction force and cycling loading applied to these systems. 


\section{Laad $(\mathrm{N})$}

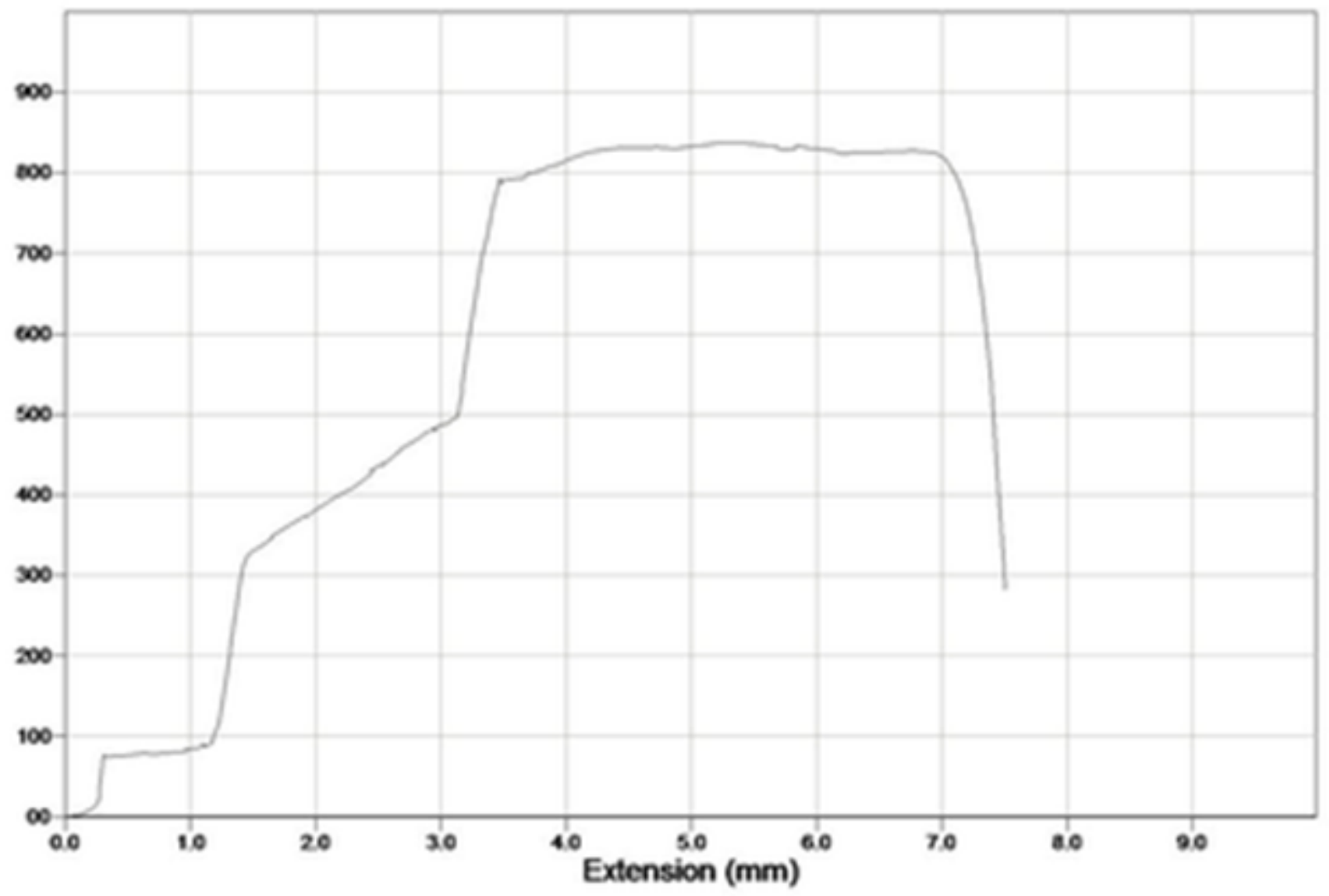

\section{Figure 4}

In group 1: increasing distraction force applied to these implants until failure. The maximum force and the total extension at the time of failure were evaluated. 
Load (N)

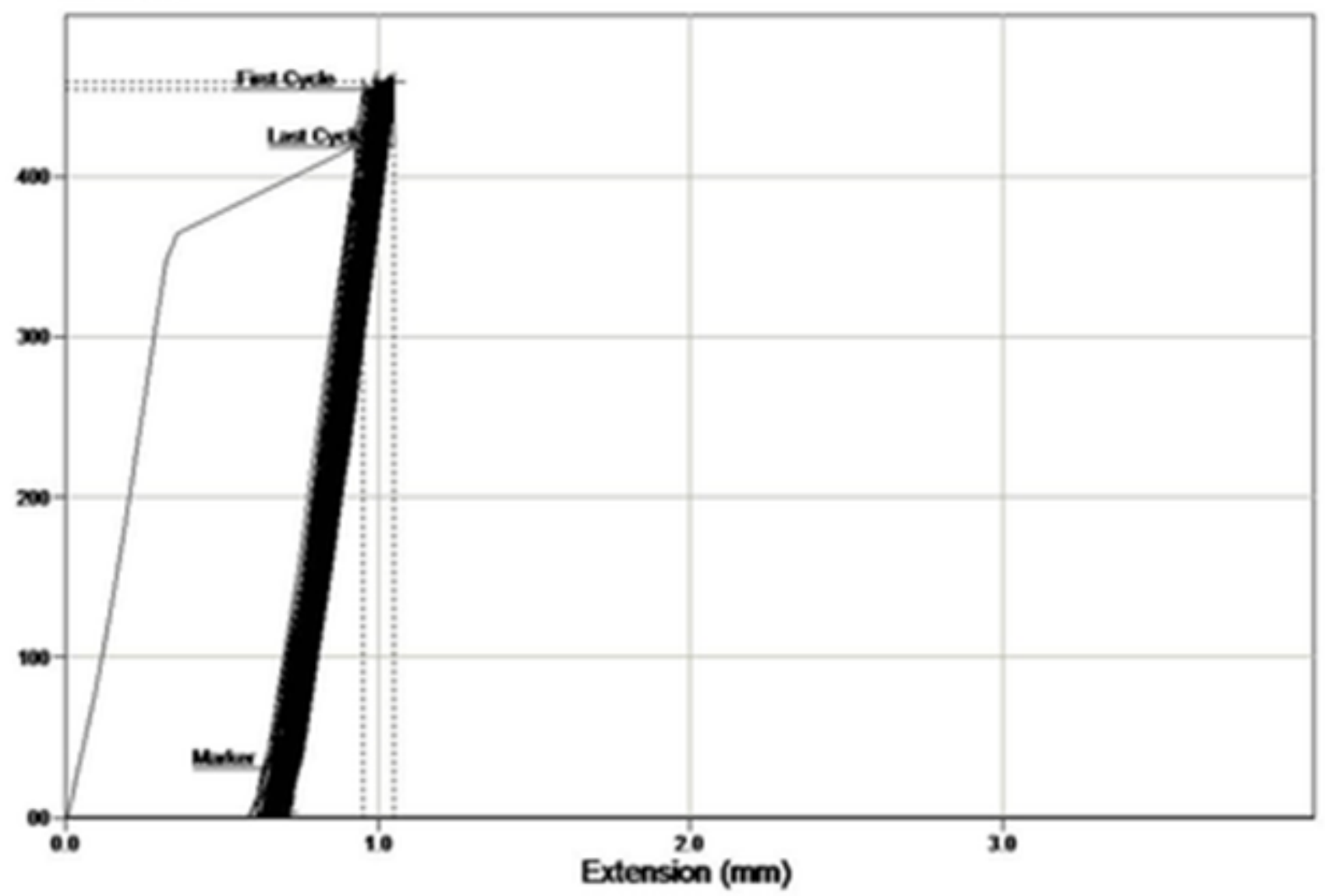

Figure 5

In group 2: to define the behavior of these EFECE implants, against cyclic loading, 450 Newton cyclic distraction forces was applied to these implants. Presence of failure and the maximum extension in a cycle were evaluated. 